Planetary Systems in the Universe - Observation, Formation and Evolution

Proceedings IAU Symposium No. 202, (C)2004 IAU

Alan Penny, Pawel Artymowicz, Anne-Marie Lagrange, \& Sara Russell, eds.

\title{
Atmospheric Circulation of Hot Jupiters
}

\author{
Tristan Guillot \\ Observatoire de la Côte d'Azur, Laboratoire Cassini, CNRS UMR 6529, \\ 06304 Nice Cedex 4, France; guillot@obs-nice.fr
}

\begin{abstract}
About $40 \%$ of the extrasolar giant planets discovered so far have orbital distances smaller than 0.2 AU. These "hot Jupiters" are expected to be in synchronous rotation with their star. The ability to measure their radii prompts a careful reexamination of their structure. I show that their atmospheric structure is complex and that thermal balance cannot be achieved through radiation only but must involve heat advection by large-scale circulation. A circulation model inspired from Venus is proposed, involving a relatively strong zonal wind (with a period that can be as short as 1 day). It is shown that even this strong wind is incapable of efficiently redistributing heat from the day side to the night side. Temperature variations of $200 \mathrm{~K}$ or more are to be expected, even at pressures as large as 10 bar. As a consequence, clouds should be absent on the day side, allowing more efficient absorption of the stellar light. The global chemical composition of the atmosphere should also be greatly affected by the presence of large temperature variations. Finally, stellar tides may also be important in their ability to deposit heat at levels untouched by stellar radiation, thereby slowing further the cooling of the planets.
\end{abstract}

Extrasolar giant planets have been known to orbit sun-like stars since the discovery of 51 Peg b by Mayor \& Queloz (1995) but an iron-clad proof of their reality had to await the recent observation of one of these planets transiting in front of its star, HD209458 (Charbonneau et al. 2000; Henry et al. 2000). The observed system consists of an F8V star and a planetary companion with a 3.524 day period and a corresponding orbital distance of 0.047 AU. Analysis of the transit observations coupled to detailed modeling of the evolution of the star yield a planetary mass of $0.69 \pm 0.05 \mathrm{M}_{\mathrm{J}}\left(\mathrm{M}_{\mathrm{J}}=1.89 \times 10^{27} \mathrm{~kg}\right.$ is the mass of Jupiter) and a radius of $1.27 \pm 0.02 \mathrm{R}_{\mathrm{J}}$ (Charbonneau et al. 2000), $1.40 \pm 0.17 \mathrm{R}_{\mathrm{J}}$ (Mazeh et al. 2000), to $1.42 \pm 0.10 \mathrm{R}_{\mathrm{J}}$ (Henry et al. 2000). $\left(\mathrm{R}_{\mathrm{J}}=70,000 \mathrm{~km}\right.$ ).

The fact that the measured radius is consistent with theoretical predictions (Guillot et al. 1996; Guillot 1999) is encouraging. It implies for example that the evolution of HD209458b is governed by the evolution of its inner radiative zone, as described in Guillot et al. $(1996 ; 1997)$, because a fully convective planet would have a radius $R \sim 3 \mathrm{R}_{\mathrm{J}}$. However, it should also be noted that the measured radius is still relatively large: Depending on factors such as age, albedo, intrinsic opacities, and equations of state, models yield radii between 1.2 and $1.7 \mathrm{R}_{\mathrm{J}}$ for a solar composition planet (Burrows et al. 2000). These radii estimates are based on atmospheric models calculated for isolated objects (Marley et al. 1996; Burrows et al. 1997), an approximation valid if the stellar flux is absorbed at deep levels. Recent atmospheric calculations which consistently include the deposition of stellar energy in the transfer equations (Seager \& Sasselov 1998; Goukenleuque et al. 2000) predict that the stellar light is absorbed 
relatively high (at pressures $\sim 0.1$ to $\sim 1$ bar), thus yielding significantly lower atmospheric temperatures (by up to $\sim 1000 \mathrm{~K}$ ). Evolution calculations based on these more detailed atmospheric models are only marginally capable of reproducing the observed radius, and allow only low amounts of heavy elements to be present in the planet, which would be somewhat surprising in regard to the significant mass-loss experienced by the planet (see Guillot 1996; Lin et al. 1996).

However, all the atmospheric models of hot Jupiters calculated thus far (Marley et al. 1996; Burrows et al. 1997; Seager \& Sasselov 1998; Marley et al. 1999; Goukenleuque et al. 2000; Sudarsky et al. 2000) are one-dimensional: they assume that globally the atmosphere can be characterized by a mean temperature profile which is the solution of the radiative transfer equations for a mean stellar flux. On the other hand, large latitudinal variations of the insolation occur. Furthermore, hot Jupiters, at orbital distances shorter than $\sim 0.2 \mathrm{AU}$, are expected to be in synchronous rotation due to the large tidal stresses raised by the star on the planet (Guillot et al. 1996). The one-dimensional approach to the radiative transfer problem can then be justified only in the presence of an efficient mechanism capable of redistributing the heat from the day side to the night side and from equator to poles.

The closest example of such an atmosphere in our Solar System is that of Venus. Being a mere 30\% closer to the Sun than its sister Earth has left Venus with a rather inhospitable $\mathrm{CO}_{2}$ atmosphere, characterized by a mean surface pressure and temperature of $95 \mathrm{bar}\left(0.95 \times 10^{7} \mathrm{~Pa}\right)$ and $740 \mathrm{~K}$. The planet rotates in the retrograde sense, with a period of 243 days, but its atmosphere has a much faster rotation period of $\sim 4$ days (in the same sense as the rotation), indicative of equatorial wind speeds of $\sim 100 \mathrm{~m} \mathrm{~s}^{-1}$ (Schubert 1983). As a consequence, both latitudinal and diurnal temperature variations remain small (i.e. within $10 \mathrm{~K}$ for $P \gtrsim 0.1$ bar) (Seiff 1983).

Can this efficient heat redistribution occur on hot Jupiters, which are characterized by an even larger insolation? For Venus, the physics that leads to superrotation is not well understood: Various mechanisms have been invoked, including gravity waves (Del Genio \& Rossow 1990), solar semi-diurnal tides (Pechmann \& Ingersoll 1984; Newman \& Leovy 1992) and barotropic instabilities produced by a Hadley cell (Gierasch 1975; Rossow \& Williams 1979). However, a key factor that qualitatively explains heat redistribution is the relatively sluggish radiative heating/cooling efficiency: below the cloud tops $(P \sim 0.1 \mathrm{bar})$, it is slower than advection by zonal and even meridional winds. Around $P \gtrsim 3$ bar, it is even slower than the length of the Venus day (117 Earth days) (Pollack \& Young 1975; Taylor et al. 1983). On hot Jupiters, the length of the day (at deep levels) is likely to be much longer because of tidal synchronization. The following discussion is based on the assumption that superrotation occurs in their atmospheres. It could for example be maintained by an upward transport of angular momentum due to the interaction between a Hadley circulation and the zonal wind, or by semi-diurnal tides. However, the timescales that are derived in this article should apply to other mechanisms. Should hot Jupiters not possess a superrotating atmosphere, slower winds would occur, thereby yielding large temperature differences between the day side and the night side (energetically, a less favorable configuration). 
Contrary to the giant planets in our solar system, the intrinsic luminosity of hot Jupiters scarcely contributes to the energy balance of their atmosphere: In the case of a Jupiter-mass planet at $0.05 \mathrm{AU}$ and after $5 \mathrm{Gyr}$ of evolution, it is expected to be similar (or slightly larger, depending on the opacities) to that of Jupiter, i.e. $L_{\text {intrinsic }} \sim 3 \times 10^{24} \mathrm{erg} \mathrm{s}^{-1}$, up to 5 orders of magnitude smaller than the received stellar luminosity. A convective instability can hence occur only in regions where the opacity is high, typically at pressures of the order of $\sim 100$ bar or more (Guillot et al. 1997). On the other hand, the structure of the atmosphere at lower pressures $(P \lesssim 10 \mathrm{bar})$ is dominated by the deposition of the stellar heat, which also yields a stable temperature profile (Seager \& Sasselov 1998; Goukenleuque et al. 2000). Necessary conditions for the Gierasch mechanism (Gierasch 1975) to occur are thus met (Del Genio et al. 1993). Figure 1 shows the expected structure of hot Jupiters, in the presence of atmospheric superrotation.

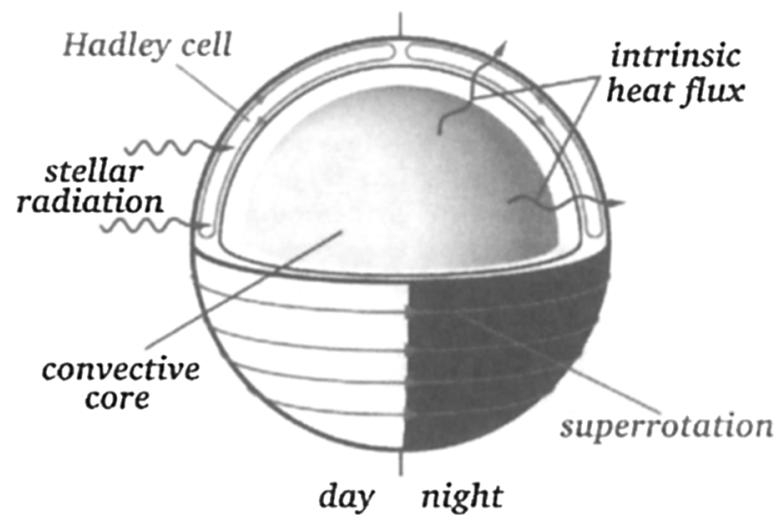

Figure 1. Conjectured dynamical structure of hot Jupiters: At pressures larger than $\sim 100$ bar, the intrinsic heat flux has to be transported by convection. The convective core is in synchronous rotation with the star and has negligible latitudinal and longitudinal temperature variations. Between $\sim 10$ and $100 \mathrm{bar}$, a relatively inert radiative layer is present. The region located at lower pressures is penetrated by the stellar light. The latitudinal variation in insolation should drive a meridional advection cell (Hadley cell), and consequently zonal winds (superrotation) propagating heat from the day side to the night side (see text).

Evidently, most of the problem then lies in estimating the strength of zonal and meridional winds compared to the ability of the atmosphere to absorb and re-radiate the stellar heat. The convective core, shielded from stellar radiation, should be locked in synchronous rotation with the star. I thus assume that no zonal winds are present $\left(u\left(P_{0}\right)=0\right)$ at this level. At higher altitudes, upward transport of angular momentum by the meridional cell yields an increase in the zonal wind intensity. The process is limited by the Kelvin-Helmotz instability that appears when the Richardson number becomes smaller than $1 / 4$ (e.g. Schubert 1983). Using the equation of state for a perfect gas, and assuming a 
uniform mean molecular weight $\mu$, one can derive a maximal zonal wind speed:

$$
u_{\max }(P) \sim \frac{1}{2} \int_{P_{0}}^{P}\left[\frac{R T}{\mu}\left(\nabla_{\mathrm{ad}}-\nabla_{T}\right)\right]^{1 / 2} d \ln p
$$

$R$ being the gas constant, $\nabla_{\mathrm{ad}}$ the adiabatic gradient, and the temperature gradient $\nabla_{T}=d \ln T / d \ln P$. The value of $u_{\max }$ thus derived is of the order of $2000 \mathrm{~m} \mathrm{~s}^{-1}$, to be compared to the strength of zonal winds in Jupiter, Saturn, Uranus and Neptune which are of the order of $100 \sim 500 \mathrm{~m} \mathrm{~s}^{-1}$ (Ingersoll et al. 1995). A characteristic timescale for zonal winds to redistribute temperature variations over scales similar to the planetary radius $R_{\text {planet }}$ then stems from $\tau_{\text {zonal }} \gtrsim R_{\text {planet }} / u_{\max }$. It can be noted that a shear instability can appear at smaller Richardson numbers in the presence of efficient radiative diffusion (Zahn 1992; Maeder 1995), but this possibility that would yield even slower zonal winds will not be examined here.

The radiative time constant is simply estimated from a ratio between the energy of a given level and its black body emission, i.e.

$$
\tau_{\text {rad }} \sim \frac{P}{g} \frac{c_{p}}{\sigma T^{3}}
$$

where $g$ is the gravity, $c_{p}$ the specific heat and $\sigma$ the Stefan-Boltzmann constant.

Figure 2 shows estimates of $\tau_{\text {zonal }}$ and $\tau_{\text {rad }}$ calculated using various temperature profiles (Burrows et al. 1997; Goukenleuque et al. 2000). Maximal uncertainties were included, for example, a temperature at 10 bar between $2100 \mathrm{~K}$ and $3500 \mathrm{~K}$. I have assumed $g=1000 \mathrm{~cm} \mathrm{~s}^{-2}, R_{\text {planet }}=1.05 \times 10^{10} \mathrm{~cm}$, $c_{p}=1.3 \times 10^{8} \mathrm{erg} \mathrm{K}^{-1} \mathrm{~g}^{-1}$ and $\nabla_{\mathrm{ad}}-\nabla_{T}=0.15$. At pressures larger than $10^{-2}$ bar, radiation is slower than the maximal advection by zonal winds, but by less than one order of magnitude. The consequent day/night temperature difference to be expected is:

$$
\Delta T / T \sim 1-e^{-\tau_{\text {zonal }} / \tau_{\text {rad }}}
$$

In the case of a planet at $0.05 \mathrm{AU}, T \sim 2000 \mathrm{~K}$ at $10 \mathrm{bar}$, therefore $\Delta T \sim 200 \mathrm{~K}$ at this level. Still higher values are likely given the fact that slower winds will lead to an even more effective cooling on the night side and heating on the day side. $\Delta T$ is also significantly larger than for Venus because of the faster radiative cooling/heating and higher temperatures.

The consequence is that hot air from the day side is cooled on the night side, where efficient condensation of cloud-forming species is to be expected. On the other hand, any condensed particle transported to the day side is expected to evaporate rapidly. Clouds should hence not form on the day side, where absorption of the stellar flux should occur at deeper levels than has been estimated (Seager \& Sasselov 1998; Goukenleuque et al. 2000). But the consequences are even more far-reaching. If vertical advection on the night side is slower than sedimentation of condensed particles, certain chemical species may be absent on the day side, at temperature and pressure at which they would be otherwise expected from thermochemical equilibrium calculations. 


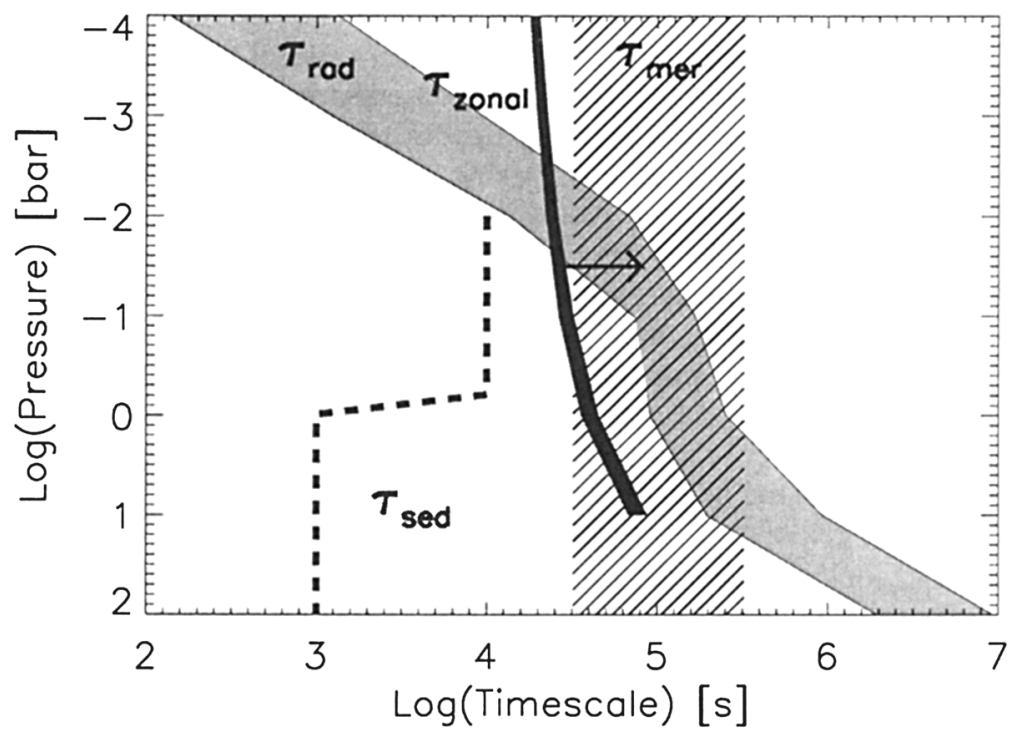

Figure 2. Characteristic time scales, as a function of pressure level: $\tau_{\text {zonal }}$ is the minimal zonal advection time. $\tau_{\text {rad }}$ is the timescale necessary to cool/heat a layer of pressure $P$ and temperature $T$ by radiation alone. $\tau_{\text {mer }}$ is the vertical meridional advection timescale. $\tau_{\text {sed }}$ is the time taken by condensing particles to grow and fall under the action of gravity, assuming that the pressure level considered is at the base of a $\mathrm{MgSiO}_{3}$ cloud in a solar-composition atmosphere. Various atmospheric models applicable to HD209458b $\left(g \sim 1000 \mathrm{~cm} \mathrm{~s}^{-2}\right.$; orbital distance $\sim 0.05 \mathrm{AU}$ ) have been used (Burrows et al. 1997; Goukenleuque et al. 2000).

The intensity of the meridional winds, $w$, can be estimated roughly estimated from a balance between radiative heating and adiabatic cooling (Gierasch 1975):

$$
w \sim \frac{\frac{g}{P_{\mathrm{rad}}} \sigma T_{\mathrm{e}}^{4}}{\frac{c_{p}}{g} T N^{2}},
$$

where $N$ is the Brunt-Väisälä frequency, $P_{\text {rad }}$ a characteristic pressure at which most of the stellar radiation is absorbed and $T_{\mathrm{e}}$ the effective temperature of the planet. The characteristic time scale associated with vertical meridional motions is then calculated as the ratio of the pressure scale height to the vertical velocity $w$ :

$$
\tau_{\mathrm{mer}} \sim \frac{c_{p} P_{\mathrm{rad}}}{g \sigma T_{\mathrm{e}}^{3}}\left(\nabla_{\mathrm{ad}}-\nabla_{T}\right)
$$

Numerical values of $P_{\mathrm{rad}}=1 \mathrm{bar}$, and $T_{\mathrm{e}}=1500 \mathrm{~K}$, lead to $\tau_{\mathrm{mer}} \sim 10^{5} \mathrm{~s}$.

The sedimentation time scale intrinsically depends on the size of the particles that are considered. Using Rossow (1978), the mean size of condensing particles can be estimated from a balance between sedimentation and growth (condensation, coagulation, coalescence). The corresponding sedimentation time scale is plotted on Fig. 2 (dashed line), in the case of a $\mathrm{MgSiO}_{3}$ cloud, assuming 
that the cloud base is at a given pressure $P$ (i.e. the temperature has been adjusted so that the ratio of the saturation vapor pressure to the total pressure is equal to the bulk abundance of the molecule). The sedimentation time scale derived is essentially proportional to the bulk abundance of the condensing species. Other significant cloud-forming species should therefore rain out on similar time scales.

Two values of $\tau_{\text {sed }}$ are found depending on whether grain growth is due to coagulation (pressures higher than $\sim 1$ bar) or condensation alone ( $P \lesssim 1$ bar). These numbers are one to more than two orders of magnitude shorter than $\tau_{\text {mer }}$, meaning that condensates forming on the night side will grow rapidly and fall before they can be transported upward. This process will therefore also efficiently remove condensing species on the day side, at levels where they are in vapor form. If a given species is predicted to condense at a pressure $P_{\text {day }}^{\star}$ and temperature $T_{\text {day }}^{\star}$ on the day side, its condensation level will be displaced on the night side according to the Clausius-Clapeyron equation:

$$
P_{\text {night }}^{\star} \sim P_{\text {day }}^{\star}\left[1+\frac{\beta \frac{\Delta T}{T_{\text {day }}^{\star}}}{\beta \nabla_{T}-1}\right],
$$

where $\beta=L / R T$ is the ratio of the latent heat of condensation $L$ to the thermal energy $R T$. Using $\beta \sim 10, \nabla_{T} \sim 0.15$ and $\Delta T / T \sim 0.1$, one finds $P_{\text {night }}^{\star} \sim 3 P_{\text {day }}^{\star}$. On the day side, the abundance of such species will be low at pressures smaller than $P_{\text {night }}^{\star}$. Furthermore, any daughter products will also be removed by this process. This is the case of $\mathrm{TiO}$, a very efficient optical absorber, which is removed by condensation of $\mathrm{CaTiO}_{3}$ (e.g. Fegley \& Lodders 1996). A number of chemical species will also be affected by this circulation mechanism, thus affecting significantly the absorption of stellar light by the planetary atmosphere. Stellar luminosity would hence be expected to penetrate deeper into the atmosphere, yielding a slower cooling and contraction of the planet.

This effect is expected to be even more pronounced for planets with a larger mass than considered here. For example, for a gravity $g=10^{4} \mathrm{~cm} \mathrm{~s}^{-2}$, both the radiative and the sedimentation timescales are decreased by a factor $\sim 10$ compared to the $g=1000 \mathrm{~cm} \mathrm{~s}^{-2}$ case. This is due to the fact that radiation is able to penetrate to larger pressure levels. Zonal rotation is expected to be relatively unaffected. This high gravity case is relevant to the recent attempt to observe the reflected light of the planetary companion to Tau Boo (Cameron et al. 1999). Although it remains very difficult to explain the large radius that is inferred for the planet using this technique (i.e. at least $1.8 \mathrm{R}_{\mathrm{J}}$, for a mass $\sim 8 \mathrm{M}_{\mathrm{J}}$, to be compared with $R \sim 1.4 \mathrm{R}_{\mathrm{J}}$ for the much less massive HD209458b), it can be advocated that a more efficient penetration of stellar light in more massive planets could slow their cooling.

Finally, it should be noted that in the presence of such an atmospheric superrotation, an equilibrium will form between atmospheric friction attempting to spin up the entire planet and the effect of tides trying to lock it into synchronous rotation (e.g. Peale \& Cassen 1978). Energy will be dissipated in the process and contribute to the energy budget of the planet, precisely in a region where the heat flux is small. It may thus contribute to further slowing down the contraction. 
Independently of the validity of the superrotation mechanism discussed here, it appears that heat cannot be completely redistributed by zonal and meridional winds in the atmospheres of synchronously rotating hot Jupiters. This conclusion should also hold for giant planets close to synchronous rotation, if they are relatively close to their star (less than, say, $0.3 \mathrm{AU}$ ). Altogether, this represents up to half of the extrasolar giant planets discovered so far. The consequent diurnal and latitudinal temperature variations should predominantly affect the evolution of hot Jupiters orbiting at less than $0.1 \mathrm{AU}$ from their star. Among all extrasolar planets, these are the most likely to be directly characterized by a number of observational techniques in the near future.

Acknowledgments. I wish to thank A. Showman, B. Gladman, M. Marley, W.B. Hubbard, C. Goukenleuque, M. Mayor and A. Maeder for a variety of useful contributions.

\section{References}

Burrows, A., Marley, M.S., Hubbard, W.B., et al. 1997, ApJ, 491, 856

Burrows, A., Guillot, T., Hubbard, W.B. et al. 2000, ApJ, 534, L97

Cameron, A.C., Horne, K., Penny, A., \& James, D. 1999, Nature, 402, 751

Charbonneau, D., Brown, T.M., Latham, D.W., \& Mayor, M. 2000, ApJ, 529, L45

Del Genio, A.D., \& Rossow, W.B. 1990, J. Atmos. Sci., 47, 293

Del Genio, A.D., Zhou, W., \& Eichler, T.P. 1993, Icarus, 101, 1

Fegley, B., Jr., \& Lodders, K. 1996, ApJ, 472, L37

Goukenleuque, C., Bézard, B., Joguet, B., Lellouch, E., \& Freedman, R. 2000, Icarus, 143,308

Gierasch, P. 1975, J. Atmos. Sci., 32, 1038

Guillot, T. 1999, Science, 286, 72

Guillot, T., Burrows, A., Hubbard, W.B., Lunine, J.I., \& Saumon, D. 1996, ApJ, 459, L35

Guillot, T., Marley, M.S., Saumon D., \& Freedman, R.S. 1997, in: Infrared Space Interferometry: Astrophysics \& the Study of Earth-like Planets, eds. C. Eiroa et al., Kluwer Academic Publishers, Dordrecht, 37

Henry, G.W., Marcy, G.W., Butler, R.P., \& Vogt, S.S. 2000, ApJ, 529, L41

Ingersoll, A.P., Barnet, C.D., Beebe, R.F. et al. 1995, in: Neptune and Triton, ed. D.P. Cruikshank, University of Arizona Press, Tucson, 613

Lin, D. N. C., Bodenheimer, P., \& Richardson, D. 1996, Nature, 380, 606

Maeder, A., 1995, A\&A, 299, 84

Marley, M.S., Saumon, D., Guillot, T., et al. 1996, Science, 272, 1919

Marley, M.S., Gelino, C., Stephens, D., Lunine, J.I., \& Freedman, R. 1999, ApJ, 513, 879

Mayor, M., \& Queloz, D. 1995, Nature, 378, 355

Mazeh, T., Naef, D., \& Torres, G. et al. 2000, ApJ, 531, in press

Newman, M., \& Leovy, C. 1992, Science, 257, 647

Peale, S.J., \& Cassen, P. 1978, Icarus, 36, 245

Pechmann, J.B., \& Ingersoll, A.P. 1984, J. Atmos. Sci., 41, 3290

Pollack, J.B., \& Young, R. 1975, J. Atmos. Sci., 32, 1025

Rossow, W.B. 1978, Icarus, 36, 1 
Rossow, W.P., \& Williams, G.P. 1979, J. Atmos. Sci., 36, 377

Schubert, G. 1983, in: Venus, eds. D.M. Hunten et al., University of Arizona Press, Tucson, 681

Seager, S., \& Sasselov, D.D. 1998, ApJ, 502, L157

Seiff, A. 1983, in: Venus, eds. D.M. Hunten et al., University of Arizona Press, Tucson, 215

Sudarksy, D., Burrows, A., \& Pinto, P. 2000, ApJ, 538, 885

Taylor, F.W., Hunten, D.M., \& Ksanfomaliti, L.V. 1983, in: Venus, eds. D.M. Hunten et al., University of Arizona Press, Tucson, 650

Zahn, J.-P. 1992, A\&A, 265, 115 